\title{
Long-term safety and effectiveness of paroxysmal atrial fibrillation ablation using a porous tip contact force-sensing catheter from the SMART SF trial
}

\author{
Andrea Natale ${ }^{1} \cdot$ George Monir ${ }^{2} \cdot$ Anshul M. Patel $^{3} \cdot$ Robert S. Fishel $^{4} \cdot$ Francis E. Marchlinski $^{5} \cdot$ M. Craig Delaughter $^{6}$. \\ Charles A. Athill ${ }^{7}$. Daniel P. Melby ${ }^{8} \cdot$ Mario D. Gonzalez ${ }^{9} \cdot$ Ramesh Hariharan $^{10} \cdot$ Brett Gidney $^{11} \cdot \operatorname{Tiffany}^{\operatorname{Tan}^{12}}{ }^{1}$ \\ Larry A. Chinitz ${ }^{13}$
}

Received: 22 January 2020 / Accepted: 11 May 2020 / Published online: 27 May 2020

(C) The Author(s) 2020

\begin{abstract}
Purpose The prospective, multicenter SMART SF trial demonstrated the acute safety and effectiveness of the 56-hole porous tip irrigated contact force $(\mathrm{CF})$ catheter for drug-refractory paroxysmal atrial fibrillation (PAF) ablation with a low primary adverse event rate (2.5\%), leading to FDA approval of the catheter. Here, we are reporting the long-term effectiveness and safety results that have not yet been reported.

Methods Ablations were performed using the 56-hole porous tip irrigated CF catheter guided by the 3D mapping system stability module. The primary effectiveness endpoint was freedom from atrial tachyarrhythmia (including atrial fibrillation, atrial tachycardia, and/or atrial flutter), based on electrocardiographic data at 12 months. Atrial tachyarrhythmia recurrence occurring 3 months post procedure, acute procedural failures such as lack of entrance block confirmation of all PVs, and undergoing repeat procedure for atrial fibrillation in the evaluation period ( 91 to 365 days post the initial ablation procedure) were considered to be effectiveness failures.

Results Seventy-eight patients (age 64.8 \pm 9.7 years; male 52.6\%; Caucasian 96.2\%) participated in the 12-month effectiveness evaluation. Mean follow-up time was $373.5 \pm 45.4$ days. The Kaplan-Meier estimate of freedom from 12-month atrial tachyarrhythmia was $74.9 \%$. Two procedure-related pericardial effusion events were reported at 92 and 180 days post procedure. There were no pulmonary vein stenosis complications or deaths reported through the 12-month follow-up period.

Conclusions The SMART SF 12-month follow-up evaluation corroborates the early safety and effectiveness success previously reported for PAF ablation with STSF.
\end{abstract}

Keywords Efficacy $\cdot$ Contact force $\cdot$ Paroxysmal atrial fibrillation $\cdot$ Porous tip $\cdot$ Radiofrequency catheter ablation $\cdot$ SmartTouch SF

\section{Introduction}

Atrial fibrillation (AF) is the most common arrhythmia diagnosed in clinical practice and the prevalence is increasing as

Andrea Natale

dr.natale@gmail.com

Texas Cardiac Arrhythmia Research Foundation, Austin, TX, USA

2 Florida Hospital Cardiovascular Research, Orlando, FL, USA

3 Emory St Joseph's Hospital, Atlanta, GA, USA

4 Florida Electrophysiology Associates, Atlantis, FL, USA

5 University of Pennsylvania, Philadelphia, PA, USA

6 HeartPlace, Bedford, TX, USA the US population ages $[1,2]$. Without treatment, AF is associated with significant morbidity and mortality, including stroke, embolism, and heart failure [3-5]. In patients with paroxysmal atrial fibrillation (PAF) refractory to drug

San Diego Cardiac Center, San Diego, CA, USA

8 Abbott Northwestern Hospital, Minneapolis, MN, USA

9 Penn-State Milton S. Hershey Medical Center, Hershey, PA, USA

10 UT Physicians - EP Heart, The Woodlands, TX, USA

11 Santa Barbara Cottage Hospital, Santa Barbara, CA, USA

12 Biosense Webster, Inc., Irvine, CA, USA

13 NYU Langone Medical Center New York University, New York, NY, USA 
treatment, radiofrequency (RF) catheter ablation is an important treatment option that has been shown to be superior to antiarrhythmic drug treatment $[6,7]$.

The THERMOCOOL SMARTTOUCH® SF Catheter (STSF), a recently developed contact force (CF) catheter, enables real-time sensing of the catheter-to-tissue contact during RF ablation [8]. A 56-hole porous tip has been incorporated in the original CF-sensing catheter design to produce more uniform cooling with less fluid delivery $[9,10]$. Compared with the traditional six-hole irrigation system, the porous tip catheter improves procedural efficiency and reduces fluid delivery $[11,12]$, while maintaining the safety profile for PAF ablation [9].

The acute data from the SMART SF study showed the STSF catheter to be safe with high early effectiveness [11]. A low primary adverse event (AE) rate of $2.5 \%(4 / 159)$ was observed, which is comparable with complication rates for traditional irrigated catheters $[7,8]$. There were no unexpected serious AEs related to the study device. Acute effectiveness, defined as confirmation of entrance block for all targeted pulmonary veins (PVs), was $96.2 \%$ for subjects who undergone RF ablation. Fluid delivered via the STSF catheter was $44.7 \%$ lower than previously reported with the traditional 6-hole nonCF irrigated THERMOCOOL $®$ catheter [7] and $52.2 \%$ lower than with the 6-hole irrigated CF THERMOCOOL SMARTTOUCH® catheter $[8,11]$.

To obtain data on long-term performance, a 12-month extension of the SMART SF study across multiple centers was conducted.

\section{Methods}

\subsection{Study design}

The SMART SF study (NCT 02359890) design was previously described. Briefly, it was a prospective, multicenter, non-randomized clinical evaluation of THERMOCOOL SMARTTOUCH® SF Catheter (STSF) for drug-refractory PAF ablation. Patient eligibility criteria in this predicate safety study included age $\geq 18$ years, symptomatic PAF with at least one documented AF episode within 1 year prior to enrollment, and a physician's note indicating a diagnosis of recurrent, selfterminating AF. Eligible patients had also previously failed at least one class I or III antiarrhythmic drug (AAD) or AV nodal blocking agent, or were intolerant to an AAD. The primary safety endpoint was met and has been previously reported [11]. The effectiveness population (EP) included patients who met all inclusion and exclusion criteria and reconsented to participate in the effectiveness phase.

The effectiveness phase of the trial, described herein, was an extension of the original safety phase that was added to evaluate 12-month effectiveness for PAF patients treated with the study catheter. The follow-up period was extended through 12 months after the initial procedure for subjects who could be re-consented. The effectiveness phase was approved by the institutional review boards or ethics committees at all participating centers and was conducted in accordance with the International Conference on Harmonization (ICH) Harmonized Tripartite Guidelines for Good Clinical Practice. To be included in the effectiveness evaluation, subjects were required to have participated in the safety phase, had ablation procedures for PAF performed with the STSF catheter, and provided consent for the extended follow-up.

The 12-month effectiveness endpoint was defined as freedom from documented AF, atrial tachycardia (AT), and/or atrial flutter (AFL) - hereinafter collectively referred to as atrial tachyarrhythmia - based on electrocardiographic data. This included ECG, telemetry strip, and 48-h Holter monitor collected at the 6-to-9- and 12-month visits during the evaluation period (91-365 days after the initial procedure). In addition, 12-month effectiveness success required freedom from acute procedural failures, which were defined as inability to confirm entrance block of all PVs at the end of ablation procedure or a use of non-study catheter for the treatment of study arrhythmia for the initial procedure. Undergoing repeat ablation for atrial tachyarrhythmia was considered an effectiveness failure.

In the effectiveness phase, all adverse events (AEs) that occurred from 30 days post-ablation through the 12-month follow-up visit were collected and reported. Procedure- or device-related serious AEs (SAEs) occurring within 30 days of the procedures were already reported in the publication of acute data [11]. For each reported AE, the severity, clinical outcome, and causality were monitored until adequately resolved or explained.

\subsection{Statistical analysis}

Descriptive statistics of demographics and baseline characteristics were calculated for patients in the EP, as well as for the original enrolled population and the subjects that did not reconsent. The 12-month effectiveness success rate was calculated by dividing the number of subjects who were free from atrial tachyarrhythmia recurrence or other effectiveness failures by the total number of subjects in the EP. The breakdown of reasons for effectiveness failures was also summarized. The success rate was compared with the performance goal of $50 \%$ using the exact test for a binomial proportion. The sample size of 78 in the EP provided at least $80 \%$ power for this test, with a one-sided significance level of 0.05 .

The Kaplan-Meier analysis with two-sided $90 \%$ confidence intervals (CI) was used to estimate the time to first atrial tachyarrhythmia recurrence. To evaluate the association between demographics, baseline medical history, and procedural data with the 12-month effectiveness endpoint, univariable and multivariable models were fit to 
Fig. 1 Patient disposition

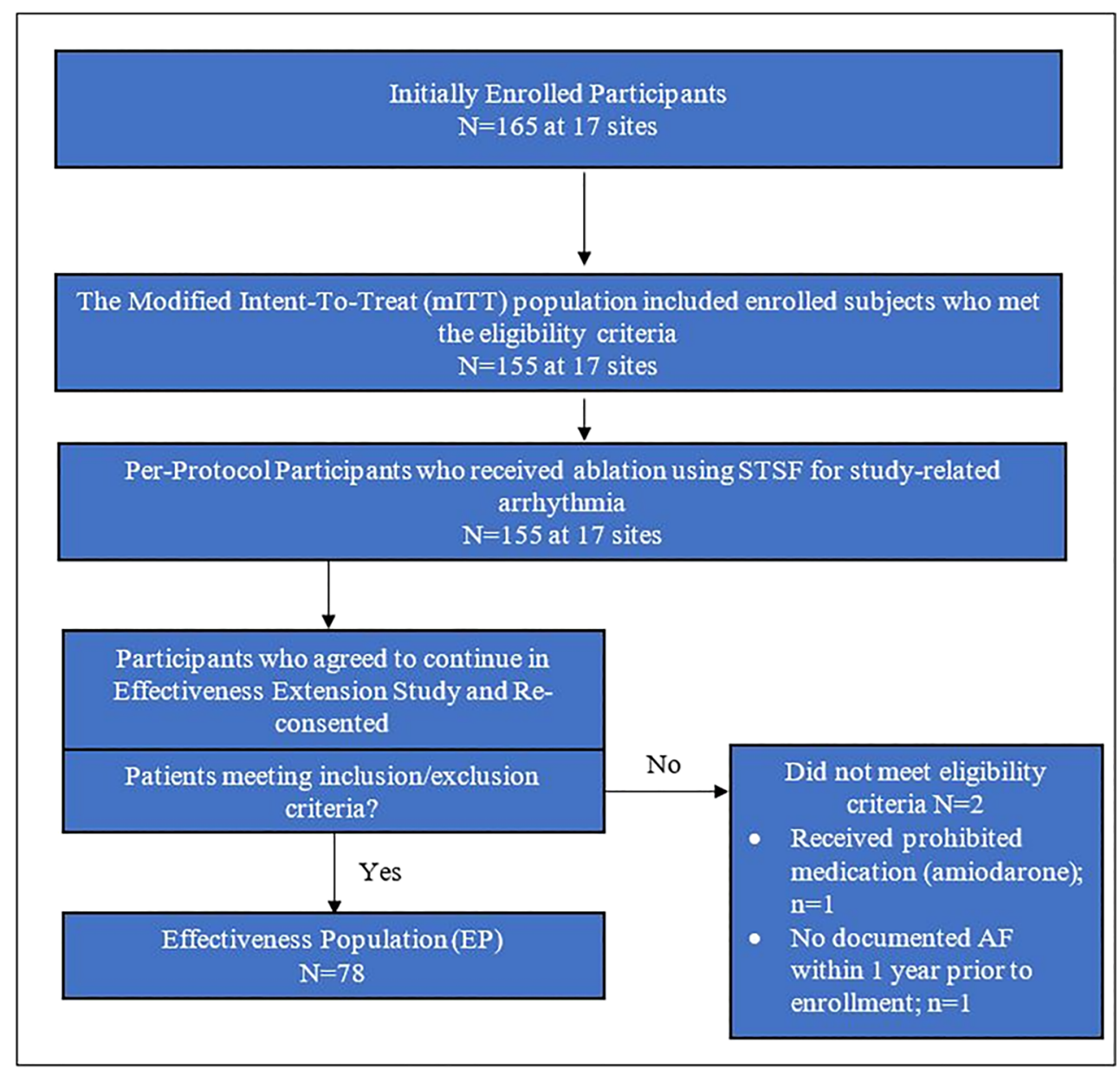

the data. If any statistically significant associations were observed at a 0.10 level, the variables were considered for the multivariable model. Multivariate logistic regression models were then used to explore the associations between key patient or procedural characteristics and the 12-month effectiveness endpoints. These models were used to estimate odds ratios (ORs) and 95\% CIs for gender, duration of AF history, previous use of class I AAD, baseline LA diameter, average contact force during ablation procedure, and the use of anticoagulation and cardiovascular medications 90 days post index procedure.

\section{Results}

\subsection{Baseline characteristics and demographics}

Eighty (80) of 135 subjects (from sites who agreed to participate in the effectiveness phase) from 13 centers re-consented to participate in the 12-month effectiveness evaluation, of which 2 were excluded because they did not meet eligibility criteria. Thus, a total of 78 patients were included in the EP.
Figure 1 displays the details of the patient disposition flow chart.

Table 1 summarizes the demographics and pre-existing baseline medical conditions for the original SMART SF trial and for the subset of patients in the EP. Overall, the EP was similar to the full patient population. In the EP, the majority of participants were white $(96.2 \%)$ and male $(52.6 \%)$, with a median age of $64.8 \pm 9.7$ years. The most common preexisting medical conditions in the EP were hypertension $(61.5 \%)$, atrial flutter $(30.8 \%)$, and coronary artery disease (20.5\%). All enrolled patients and patients in the EP experienced symptomatic AF for an average of 47.9 and 41.3 months prior to enrollment, respectively. The median duration from patients' initial AF diagnosis to enrollment was 24.0 months (all enrolled) and 19.5 months (EP). The mean follow-up duration for the 78 patients was $373.5 \pm 45.38$ days from index procedure. Compared with those who elected not to be included in the study extension through 12 months of follow-up (non-EP), patients in the EP $(n=78)$ had a slightly higher proportion of female participants $(47.4 \%$ vs. $32.5 \%)$, higher mean age (64.8 vs. 60.5 years), higher proportion of patients with coronary artery disease $(20.5 \%$ vs. $15.6 \%)$, and more symptomatic AF at baseline. 
Table 1 Baseline demographic and patient characteristics

\begin{tabular}{|c|c|c|c|}
\hline Characteristics & $\begin{array}{l}\text { Enrolled } \\
n=165^{*}\end{array}$ & $\begin{array}{l}\text { EP } \\
n=78\end{array}$ & $\begin{array}{l}\text { Non-EP } \\
n=77\end{array}$ \\
\hline Age, mean $\pm \mathrm{SD}$, years & $62.7 \pm 10.4$ & $64.8 \pm 9.7$ & $60.5 \pm 10.2$ \\
\hline Male & $95(57.9)$ & $41(52.6)$ & $52(67.5)$ \\
\hline Race, Caucasian & $159(97.0)$ & $75(96.2)$ & $76(98.7)$ \\
\hline \multicolumn{4}{|l|}{ Pre-existing baseline medical condition(s)* } \\
\hline Coronary artery disease & $29(17.8)$ & $16(20.5)$ & $12(15.6)$ \\
\hline Congestive heart failure & $6(3.7)$ & $1(1.3)$ & $4(5.2)$ \\
\hline Myocardial infarction & $6(3.7)$ & $1(1.3)$ & $5(6.5)$ \\
\hline Significant valve disease & $3(1.8)$ & $2(2.6)$ & $1(1.3)$ \\
\hline Thromboembolic event & $10(6.1)$ & $7(9.0)$ & $3(3.9)$ \\
\hline Atrial flutter & $51(31.3)$ & $24(30.8)$ & $23(29.9)$ \\
\hline Diabetes & $23(14.1)$ & $12(15.4)$ & $10(13.0)$ \\
\hline Hypertension & $93(57.1)$ & $48(61.5)$ & $42(54.5)$ \\
\hline \multicolumn{4}{|l|}{ Baseline transthoracic echocardiogram (TTE) } \\
\hline Mean left ventricle ejection fraction (\%) & 60.1 & 59.0 & 60.8 \\
\hline Mean LA diameter from PLAX (mm) & 38.8 & 38.4 & 39.3 \\
\hline \multicolumn{4}{|l|}{ AF history } \\
\hline Mean $\mathrm{AF}$ duration, months prior to enrollment & 47.9 & 41.3 & 50.8 \\
\hline Median AF duration, months prior to enrollment & 24.0 & 19.5 & 24.0 \\
\hline \multicolumn{4}{|l|}{ AF symptoms } \\
\hline Chest pain & $16(9.8)$ & $10(12.8)$ & $6(7.79)$ \\
\hline Dizziness & $46(28.2)$ & $23(29.5)$ & $21(27.27)$ \\
\hline Dyspnea & $69(42.3)$ & $34(43.6)$ & $35(45.45)$ \\
\hline Palpitations & $129(79.1)$ & $58(74.4)$ & $64(83.12)$ \\
\hline Syncope & $9(5.5)$ & $5(6.4)$ & $3(3.90)$ \\
\hline Weakness & $30(18.4)$ & $21(26.9)$ & $9(11.69)$ \\
\hline Nausea & $9(5.5)$ & $7(9.0)$ & $2(2.60)$ \\
\hline Lightheadedness & $46(28.2)$ & 27 (34.6) & 19 (24.68) \\
\hline Fatigue & $84(51.5)$ & $41(52.6)$ & $38(49.35)$ \\
\hline
\end{tabular}

Values are $n(\%)$ unless specified

$E P$, effectiveness population; $S D$, standard deviation; $P L A X$, parasternal long axis

*Pre-existing baseline medical conditions, AF history, and AF symptoms were summarized based on 163 enrolled subjects who have available medical history and AF baseline data

During the effectiveness phase of the study, the baseline medical history case report form was unlocked for patients enrolled in this portion of the study, which resulted in including the identification of other arrhythmias such as premature ventricular contractions and atrial tachycardia

\subsection{Effectiveness}

The Kaplan-Meier estimate of freedom from atrial tachyarrhythmia recurrence 12 months was $74.9 \%$ (90\% CI [65.0\%, $84.7 \%]$ ) with 12 subjects experiencing recurrence of atrial fibrillation. The lower bound of the $90 \%$ confidence interval was greater than the pre-determined performance goal of 50\%; thus, the effectiveness endpoint was met (Fig. 2). Table 2 summarizes the reasons for the 19 patients who failed the 12-month effectiveness criteria.

Using a univariate model, gender, duration of AF history, previous class I AAD history, baseline LA dimension, average contact force, and post-AF cardiac medications were identified as predictors of failure in regard to 12-month failure (Table 3). Using a multivariate logistic regression model, class I AAD history was a significant predictor of failure at $p \leq 0.05$ (OR 5.3, 95\% CI [1.32, 21.55]). Females tended to have lower 12-month success rate compared with males with $\mathrm{OR}=3$ (95\% CI 0.79, 11.27). An additional month in duration of AF history increased the odds of failure by $1 \%$. Patients with larger baseline LA dimension, higher contact force, and postAF cardiac medications were less likely to fail (OR and 95\% CI 0.94 [0.84, 1.05], 0.88 [0.74, 1.05], 0.34 [0.08, 1.38], respectively). 
Fig. 2 Kaplan-Meier freedom from tachyarrhythmia (effectiveness population, $N=78$ )

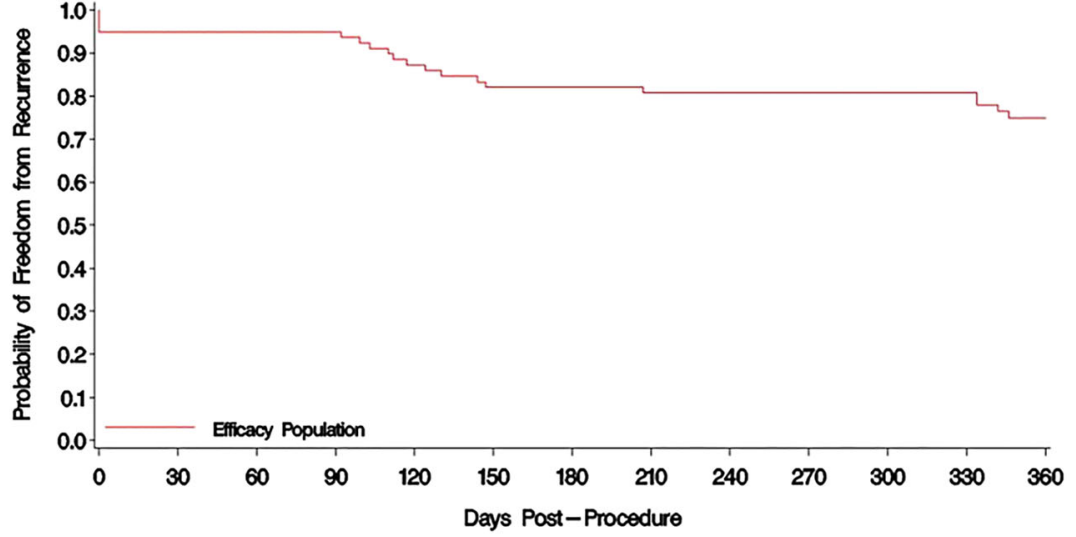

Number of subjects at risk at the associated timepoints

\begin{tabular}{lccccccccccccc}
\hline & 0 & 30 & 60 & 90 & 120 & 150 & 180 & 210 & 240 & 270 & 300 & 330 & 360 \\
$\begin{array}{l}\text { Days Post-Procedure } \\
\begin{array}{l}\text { Number of subjects } \\
\text { at risk }\end{array}\end{array}$ & 78 & 74 & 74 & 74 & 74 & 68 & 64 & 64 & 63 & 62 & 62 & 61 & 58 \\
\hline
\end{tabular}

\subsection{Safety}

From 30-day post-ablation procedure through the 12-month follow-up, there were a total of 15 serious AEs (SAEs) that occurred in 6 patients. All SAEs were considered unrelated to the study device by the investigators. One pericardial effusion, discovered at 92 days post procedure, was classified as serious and related to the procedure. A second pericardial effusion occurred at 180 days post procedure and was classified as possibly procedure related. There were no events of pulmonary vein stenosis and no deaths reported through the effectiveness follow-up period.

\section{Discussion}

This prospective multicenter study has demonstrated safety and efficacy of STSF for the treatment of patients with drug-

Table 2 12-month effectiveness (effectiveness population, $N=78$ )

\begin{tabular}{ll}
\hline Primary outcome and reasons for failure & $n(\%)$ \\
\hline Total successes & $59 / 78(75.6 \%)$ \\
Total failures & $19 / 78(24.4 \%)$ \\
AF/AT/AFL recurrence* & $12(15.4 \%)$ \\
AT & $2(2.6 \%)$ \\
AF & $10(12.8 \%)$ \\
Acute failure** & $4(5.1 \%)$ \\
Repeat ablation post-blanking period & $3(3.8 \%)$
\end{tabular}

*AF/AT/AFL $=$ atrial fibrillation/atrial tachycardia/atrial flutter

**Acute failure is defined as lack of entrance block confirmation for all PV refractory symptomatic PAF. The Kaplan-Meier estimate of 12-month effectiveness success was $74.9 \%$ with a low incidence of SAEs $(7.7 \%)$ reported between days 30 and 360, the majority $(87 \%)$ of which were unrelated to the device/ procedures.

Compared with traditional 6-hole contact force-sensing catheters, the THERMOCOOL SMARTTOUCH® SF catheter has a 56-hole porous tip and delivers uniform cooling at half the flow rate. This prior study showed that the reduction in flow rate resulted in an overall decrease in fluid delivery of 44.7 to $52.2 \%$, a significant consideration to patients with existing comorbidities, in which fluid overload could adversely affect cardiac function. The positive safety and short-term effectiveness observations in the initial safety phase presented in Chinitz et al. [11] were also associated with improved procedural efficiency with respect to procedure and fluoroscopy times.

Reports of the long-term outcomes of AF ablation with STSF are limited. Previous studies reported 12-month success rates between 80 and $90 \%$ in PAF populations (including short-term persistent AF in one study) from single-center or limited multicenter evaluations $[12,13]$. Our results represent the experience of a large number of centers (13), therefore likely representative of broader real-world practice. Overall, 12-month effectiveness with STSF was comparable with a previous PAF ablation study (SMART AF) of similar study design using the predecessor 6-hole irrigated CF catheters [8]. Compared with the original THERMOCOOL AF IDE study, conducted almost a decade ago, the current success rates in the SMART AF and SMART SF studies are substantially higher (74-75\% vs. 66\%) [7]. This finding is further supported by a previous meta-analysis across $\mathrm{CF}$ vs non-CF studies showing a decrease in 12-month AF recurrence with the use of $\mathrm{CF}$ technology [14-16]. The upward trend in long-term success 
Table 3 Multivariate logistic regression for failure of 12-month primary effectiveness endpoint in the EP population $(N=78$ (the regression model included 72 of the 78 subjects due to 2 subjects missing baseline LA dimension and 4 subjects missing contact force measurement))

\begin{tabular}{llll}
\hline Predictors & & Odds ratio & 95\% Wald CI \\
\hline Gender & Female vs. male & 2.98 & $(0.79,11.27)$ \\
Duration of AF history (months) & & 1.01 & $(1.00,1.02)$ \\
Previous AAD: class I** & Yes vs. no & 5.32 & $(1.32,21.55)$ \\
Baseline LA dimension & & 0.94 & $(0.84,1.05)$ \\
Average contact force & & 0.88 & $(0.74,1.05)$ \\
Post-AF cardiac medications & Yes vs. no & 0.34 & $(0.08,1.38)$ \\
\hline
\end{tabular}

**Predictor statistically significant at 0.05 level over time and over $\mathrm{CF}$ vs. non-CF ablation studies is encouraging and consistent with the expectation of improved outcomes due to advancement in technologies and catheter ablation experience. Safety of PAF ablation with STSF is further confirmed from long-term safety monitoring of this trial. More recently, a real-world multicenter Italian registry utilizing STSF/Visitag reported higher 12-month success of $90 \%$ with lower procedure time (100 $\mathrm{min})$ and fluoroscopy time $(6 \mathrm{~min})$ [13].

Compared with those who elected not to be included in the study extension through 12 months of follow-up (non-EP), patients in the EP group tended to have higher percentage of female patients and higher proportion with coronary artery disease. This is reflective of real-world patient decisionmaking in that patients with symptoms are more likely to seek continue treatment in contrast to patients who feel better who are more likely to exit treatment follow-up. In addition, both gender (being female) and advanced age have previously been shown to be risk factors for recurrence after catheter ablation [17-19]. It is possible that the 12-month success rate observed in the effectiveness evaluation period of this study represented a more symptomatic or sicker patient population than that of the original study, and thus that the success rate in a more representative population would have been higher than what was observed.

This study is limited by the single-arm design and the need for patients to re-consent in order to continue in the effectiveness evaluation phase. Of the 165 patients in the initial safety study, 135 were invited to participate in the effectiveness phase of the study and 80 consented to continue in the study. As such, study participation was not randomized and could have been biased, as observed with some differences in baseline patient characteristics and comorbidities between EP and non-EP patients.

\section{Conclusion}

The SMART SF 12-month evaluation confirms the effectiveness of PAF ablation with STSF. One-year success is high (74.9\%) and is coupled with a good long-term safety profile.
Acknowledgments We wish to express our appreciation to all SMART SF trial study investigators and the following individuals for their contribution to the statistical analysis and editorial assistance (including collecting authors' comments, copyediting, and referencing) with the manuscript: Sidan He, Maciej Gonek, and Lee Ming Boo. Additional editorial support including formatting was provided by CTI Clinical Trial Services, and funded by Biosense Webster, Inc. The authors retained full control of manuscript content development.

Funding information The study was sponsored by Biosense Webster, Inc.

Open Access This article is licensed under a Creative Commons Attribution 4.0 International License, which permits use, sharing, adaptation, distribution and reproduction in any medium or format, as long as you give appropriate credit to the original author(s) and the source, provide a link to the Creative Commons licence, and indicate if changes were made. The images or other third party material in this article are included in the article's Creative Commons licence, unless indicated otherwise in a credit line to the material. If material is not included in the article's Creative Commons licence and your intended use is not permitted by statutory regulation or exceeds the permitted use, you will need to obtain permission directly from the copyright holder. To view a copy of this licence, visit http://creativecommons.org/licenses/by/4.0/.

\section{References}

1. Morillo CA, Banerjee A, Perel P, Wood D, Jouven X. Atrial fibrillation: the current epidemic. Journal of geriatric cardiology. JGC. 2017;14(3):195-203. https://doi.org/10.11909/j.issn.1671-5411. 2017.03.011.

2. Chugh SS, Havmoeller R, Narayanan K, Singh D, Rienstra M, Benjamin EJ, et al. Worldwide epidemiology of atrial fibrillation: a Global Burden of Disease 2010 Study. Circulation. 2014;129(8): 837-47. https://doi.org/10.1161/CIRCULATIONAHA.113. 005119.

3. Benjamin EJ, Wolf PA, D'Agostino RB, Silbershatz H, Kannel WB, Levy D. Impact of atrial fibrillation on the risk of death: the Framingham Heart Study. Circulation. 1998;98(10):946-52.

4. Wolf PA, Dawber TR, Thomas HE Jr, Kannel WB. Epidemiologic assessment of chronic atrial fibrillation and risk of stroke: the Framingham study. Neurology. 1978;28(10):973-7.

5. Yaghi S, Kamel H. Stratifying stroke risk in atrial fibrillation: beyond clinical risk scores. Stroke. 2017;48(10):2665-70. https://doi. org/10.1161/STROKEAHA.117.017084.

6. Calkins H, Kuck KH, Cappato R, Brugada J, Camm AJ, Chen SA, et al. 2012 HRS/EHRA/ECAS Expert Consensus Statement on Catheter and Surgical Ablation of Atrial Fibrillation: 
recommendations for patient selection, procedural techniques, patient management and follow-up, definitions, endpoints, and research trial design. Europace. 2012;14(4):528-606. https://doi. org/10.1093/europace/eus027.

7. Wilber DJ, Pappone C, Neuzil P, De Paola A, Marchlinski F, Natale $\mathrm{A}$, et al. Comparison of antiarrhythmic drug therapy and radiofrequency catheter ablation in patients with paroxysmal atrial fibrillation: a randomized controlled trial. Jama. 2010;303(4):333-40. https://doi.org/10.1001/jama.2009.2029.

8. Natale A, Reddy VY, Monir G, Wilber DJ, Lindsay BD, McElderry HT, et al. Paroxysmal AF catheter ablation with a contact force sensing catheter: results of the prospective, multicenter SMARTAF trial. J Am Coll Cardiol. 2014;64(7):647-56. https://doi.org/10. 1016/j.jacc.2014.04.072.

9. Bertaglia E, Fassini G, Anselmino M, Stabile G, Grandinetti G, De Simone A, et al. Comparison of ThermoCool(R) Surround Flow catheter versus ThermoCool(R) catheter in achieving persistent electrical isolation of pulmonary veins: a pilot study. J Cardiovasc Electrophysiol. 2013;24(3):269-73. https://doi.org/10.1111/jce. 12031.

10. Park CI, Lehrmann H, Keyl C, Weber R, Schurr P, SchiebelingRomer J, et al. Enhanced efficiency of a novel porous tip irrigated $\mathrm{RF}$ ablation catheter for pulmonary vein isolation. J Cardiovasc Electrophysiol. 2013;24(12):1328-35. https://doi.org/10.1111/jce. 12221.

11. Chinitz LA, Melby DP, Marchlinski FE, Delaughter C, Fishel RS, Monir G, et al. Safety and efficiency of porous-tip contact-force catheter for drug-refractory symptomatic paroxysmal atrial fibrillation ablation: results from the SMART SF trial. Europace. 2017;20(3):392-400. https://doi.org/10.1093/europace/eux264.

12. Maurer T, Rottner L, Makimoto H, Reissmann B, Heeger CH, Lemes $\mathrm{C}$, et al. The best of two worlds? Pulmonary vein isolation using a novel radiofrequency ablation catheter incorporating contact force sensing technology and 56-hole porous tip irrigation. Clin Res Cardiol. 2018;107:1003-12. https://doi.org/10.1007/s00392018-1270-y.
13. Stabile G, Di Donna P, Schillaci V, Di Monaco A, Iuliano A, Caponi D, et al. Safety and efficacy of pulmonary vein isolation using a surround flow catheter with contact force measurement capabilities: a multicenter registry. J Cardiovasc Electrophysiol. 2017;28(7):762-7. https://doi.org/10.1111/jce.13227.

14. Afzal MR, Chatta J, Samanta A, Waheed S, Mahmoudi M, Vukas $\mathrm{R}$, et al. Use of contact force sensing technology during radiofrequency ablation reduces recurrence of atrial fibrillation: a systematic review and meta-analysis. Heart Rhythm. 2015;12(9):1990-6. https://doi.org/10.1016/j.hrthm.2015.06.026.

15. Macle L, Frame D, Gache LM, Monir G, Pollak SJ, Boo LM. Atrial fibrillation ablation with a spring sensor-irrigated contact forcesensing catheter compared with other ablation catheters: systematic literature review and meta-analysis. BMJ Open. 2019;9(6): e023775. https://doi.org/10.1136/bmjopen-2018-023775.

16. Zhou X, Lv W, Zhang W, Ye Y, Li Y, Zhou Q, et al. Impact of contact force technology on reducing the recurrence and major complications of atrial fibrillation ablation: a systematic review and meta-analysis. Anatol J Cardiol. 2017;17(2):82-91. https:// doi.org/10.14744/AnatolJCardiol.2016.7512.

17. Takigawa M, Kuwahara T, Takahashi A, Watari Y, Okubo K, Takahashi Y, et al. Differences in catheter ablation of paroxysmal atrial fibrillation between males and females. Int $\mathrm{J}$ Cardiol. 2013;168(3):1984-91. https://doi.org/10.1016/j.ijcard.2012.12. 101.

18. Zhang XD, Gu J, Jiang WF, Zhao L, Wang YL, Liu YG, et al. The impact of age on the efficacy and safety of catheter ablation for long-standing persistent atrial fibrillation. Int $\mathrm{J}$ Cardiol. 2013;168(3):2693-8. https://doi.org/10.1016/j.ijcard.2013.03.025.

19. Zhang XD, Tan HW, Gu J, Jiang WF, Zhao L, Wang YL, et al. Efficacy and safety of catheter ablation for long-standing persistent atrial fibrillation in women. Pacing Clin Electrophysiol. 2013;36(10):1236-44. https://doi.org/10.1111/pace.12212.

Publisher's note Springer Nature remains neutral with regard to jurisdictional claims in published maps and institutional affiliations. 